\title{
High heritability for Ascaris and Trichuris infection levels in pigs
}

\author{
P Nejsum ${ }^{1,2}$, A Roepstorff ${ }^{1}$, CB Jørgensen², M Fredholm² ${ }^{2}$ HHH Göring ${ }^{3}$, TJC Anderson ${ }^{3}$ \\ and SM Thamsborg ${ }^{1}$ \\ ${ }^{1}$ Danish Centre for Experimental Parasitology, Department of Veterinary Pathobiology, Faculty of Life Sciences, University of \\ Copenhagen, Copenhagen, Denmark; ${ }^{2}$ Genetics and Bioinformatics, Department of Animal and Veterinary Basic Sciences, Faculty of Life \\ Sciences, University of Copenhagen, Copenhagen, Denmark and ${ }^{3}$ Department of Genetics, Southwest Foundation for Biomedical \\ Research, San Antonio, TX, USA
}

\begin{abstract}
Aggregated distributions of macroparasites within their host populations are characteristic of most natural and experimental infections. We designed this study to measure the amount of variation that is attributable to host genetic factors in a pig-helminth system. In total, 195 piglets were produced after artificial insemination of 19 sows (Danish LandraceYorkshire crossbreds) with semen selected from 13 individual Duroc boars (1 or 2 sows per boar; mean litter size: 10.3; 5-14 piglets per litter). Starting at 10 weeks of age, piglets were repeatedly infected with the gastrointestinal helminths Trichuris suis and Ascaris suum by administering eggs in the feed for 14 weeks until necropsy. Faecal egg counts (FECs) were estimated regularly and $A$. suum worm burden was obtained at necropsy. Heritability calculations for $\log ($ FEC +1$)$ at weeks $7-10$ post-infection (p.i.) showed that
\end{abstract}

$0.32-0.73$ of the phenotypic variation for $T$. suis could be attributed to genetic factors. For $A$. suum, heritabilities of $0.29-0.31$ were estimated for $\log (F E C+1)$ at weeks $7-14$ p.i., whereas the heritability of log worm counts was 0.45 . Strong positive genetic correlations $(0.75-0.89)$ between $T$. suis and $A$. suum FECs suggest that resistance to both infections involves regulation by overlapping genes. Our data demonstrate that there is a strong genetic component in resistance to $A$. suum and $T$. suis infections in pigs. Identification of responsible genes would enhance our understanding of the host immune response to these common nematodes and for the closely related species ( $T$. trichiura and A. lumbricoides) in man infecting more than a billion people. Heredity (2009) 102, 357-364; doi:10.1038/hdy.2008.131; published online 14 January 2009

Keywords: Ascaris suum; Trichuris suis; heritability; host genotypes; resistance; breeding

\section{Introduction}

Helminth infections are typically overdispersed (aggregated) within the host population, with a minority of the population harbouring the majority of the worm load (for example, Anderson and May, 1985). For example, in both natural and experimental (single and trickle) Ascaris suum infections of pigs, a typical aggregated distribution of parasites within the host population has been shown (reviewed by Roepstorff, 2003), with around $20 \%$ of the host population harbouring $80 \%$ of the parasites. Furthermore, 'wormy' pigs seem to be predisposed to high infection levels, as they become reinfected to a higher extent than their less wormy penmates after an anthelmintic treatment (Boes et al., 1998b). In single infections, the aggregated distribution is the result of expulsion of high numbers of larvae from the small intestine during the early phase of infection where after only few pigs are worm positive (Roepstorff et al., 1997). Results from a low number of trickle-infected pigs

Correspondence: Dr P Nejsum, Department of Veterinary Pathobiology, Faculty of Life Sciences, Danish Centre for Experimental Parasitology, Copenhagen University, Dyrlaegevej 100, DK-1870, Frederiksberg C, Copenhagen, Denmark.

E-mail:pn@life.ku.dk

Received 7 May 2008; revised 2 September 2008; accepted 7 December 2008; published online 14 January 2009 indicate the same (Eriksen et al., 1992). In these experimental infections, a number of potential 'aggregation factors' such as different exposure/behaviour, uneven level of acquired resistance prior to infection, sex, age, breed and infection doses were controlled, which suggest a regulating role of genetic factors on worm load. These factors may very likely be associated with host genetics. In humans, Williams-Blangero et al. (1999) found strong support for genetic factors accounting for 30-50\% of the variation in A. lumbricoides infection levels in a Nepalese population. Three significant and three suggestive quantitative trait loci influencing susceptibility to $A$. lumbricoides infection have now been identified (Williams-Blangero et al., 2008a).

For Trichuris suis, we are not aware of any published studies focusing on the effect of host genetics on infection levels, but several studies have shown familial or household effects on T. trichiura infections in human populations (Forrester et al., 1990; Anderson et al., 1993; Chan et al., 1994a, b; Ellis et al., 2007). Recently, WilliamsBlangero et al. (2002) have been able to disentangle the genetic effects on $T$. trichiura infection levels (as measured by faecal egg counts (FECs)) in two Asian populations. Approximately $28 \%$ of the variation within these two populations could be attributed to genetic factors, whereas household effects only accounted for $4 \%$. This finding strongly suggests that susceptibility to 
T. trichiura has a host genetic component, which recently has been supported by localization of two significant quantitative trait loci in one of these two populations (Williams-Blangero et al., 2008b).

Genetic markers with influence on helminth resistance in ruminants have been identified (for example, Sayers et al., 2005) as have markers for Escherichia coli resistance in pigs (Meijerink et al., 1997; Jørgensen et al., 2004). In recent years, marker-assisted selection has received increased attention as this approach allows the selection of animals without producing the phenotype (Dekkers, 2004). In human populations, identification of the genetic factors underlying a specific phenotype such as resistance to pathogens is challenging because populations are heterogeneous in age, exposure to infection, nutritional and immune status and many other factors. In contrast, domestic animals, such as pigs constitute a more experimentally tractable resource for understanding the genetic basis of phenotypic variation (for example, Milan et al. 2000; Van Laere et al., 2003). In animal studies, it is possible to establish 'ideal' pedigrees (resource families) in which specific traits can be predicted to segregate and family sizes are typically higher than in humans, which improve the power to detect heritable traits. Most importantly, controlled infections of pigs can be conducted and environmental variation can be minimized - as such we are able to examine infection dynamics at defined intervals following infection.

The present study aims at assessing the contribution of host genetic factors in determining infection outcomes in pigs exposed to infection with A. suum and T. suis. To do this, we used controlled trickle infections, which mimic natural transmission, in a resource population with known pedigree.

\section{Materials and methods}

\section{Inoculation material}

A. suum eggs (DCEP batch 03-02) were isolated as described by Oksanen et al. (1990) from A. suum females collected at a local abattoir and stored in $0.05 \mathrm{M} \mathrm{H}_{2} \mathrm{SO}_{4}$ with less than 20 eggs $\mu \mathrm{l}^{-1}$. The infectivity of the batch was tested by inoculating each of two pigs (10 weeks of age) by stomach tube with 1000 embryonated eggs. The pigs were killed at day 12 post-infection (p.i.) and A. suum larvae were isolated from the small intestine and enumerated. Recovery rates were 38 and $62 \%$. The T. suis egg batch, originally isolated from an organic farm (Roepstorff and Murrell, 1997), had an infectivity rate of approximately 100\%, as described by Kringel and Roepstorff (2006).

\section{The resource population}

From two commercial specific pathogen-free farms, 111 and 84 weaned piglets were obtained from 10 and 9 sows, respectively (Table 1), with an overall mean litter size at 10.3 (minimum-maximum: 5-14). The piglets were the outcome of artificial insemination of 19 Danish Landrace-Yorkshire crossbred sows with individual semen from 13 Duroc boars. The pigs were produced in two rounds 2 weeks apart (8 and 11 litters, respectively). All pigs were ear-tagged and males were castrated.
Table 1 Numbers of piglets produced on two farms by inseminating sows with semen from specific boars

\begin{tabular}{ccccc}
\hline Sow & Boar & Farm & Delivery & No. of pigs \\
\hline 1 & 1 & 1 & 2 & 8 \\
2 & 1 & 2 & 2 & 10 \\
3 & 2 & 1 & 1 & 13 \\
4 & 2 & 2 & 2 & 8 \\
5 & 3 & 1 & 1 & 13 \\
6 & 3 & 2 & 2 & 11 \\
7 & 4 & 1 & 1 & 13 \\
8 & 4 & 2 & 2 & 10 \\
9 & 5 & 1 & 2 & 11 \\
10 & 5 & 2 & 2 & 8 \\
11 & 6 & 1 & 1 & 8 \\
12 & 6 & 2 & 2 & 5 \\
13 & 7 & 1 & 1 & 14 \\
14 & 8 & 1 & 1 & 9 \\
15 & 9 & 1 & 1 & 12 \\
16 & 10 & 1 & 2 & 10 \\
17 & 11 & 2 & 2 & 9 \\
18 & 12 & 2 & 2 & 13 \\
19 & 13 & 2 & 2 & \\
\hline
\end{tabular}

\section{Experimental design and laboratory analysis}

The infection study was performed under outdoor conditions at the research farm of Copenhagen University during wintertime to avoid autoinfection (Larsen and Roepstorff, 1999). At 8 weeks of age, the pigs were randomly allocated, after stratification according to farm, sex and litter, to six helminth-free paddocks of equal size. The pigs from the two deliveries (2 weeks in between) were allocated into two and four paddocks, respectively. The pigs were fed a standard diet consisting of ground barley with protein/mineral supplement and had free access to water. After 2 weeks of acclimatization, the pigs received $A$. suum and $T$. suis trickle infection (25 and 5 eggs kg ${ }^{-1}$ day $^{-1}$, respectively) in the feed twice weekly until slaughter at week 14 post start of infection (p.i.). The dose was adjusted weekly on a pen level according to mean weight of the pigs. Faecal samples were taken from each pig at weeks $0,6,7,8,9,10,12$ and 14 p.i. and FECs were determined by a modified McMaster technique with saturated $\mathrm{NaCl}$ with $500 \mathrm{~g}$ glucose per litre (specific gravity $1.27 \mathrm{~g} \mathrm{ml}^{-1}$ ), with a lower detection limit of 20 eggs per gram of faeces (Roepstorff and Murrell, 1998). False-positive egg counts due to coprophagia have been shown for A. suum and T. suis (Boes et al., 1997, 1998a) and an arbitrary cutoffs at FEC $<41$ and $<21$ for A. suum and T. suis, respectively, were applied to define non-infected pigs for the prevalences and frequency distributions of worm load, whereas heritability calculations were based on raw data. Pig body weights were obtained at weeks 0,7 and 14 p.i. At slaughter, the pigs were exviscerated and the small intestine was cut open. The contents were sieved and $A$. suum visible by the naked eye were recovered and stored in $70 \%$ ethanol. All worms $(n=4758)$ were subsequently sexed, length measured, weighed and enumerated. Actual worm counts for $T$. suis were not determined as very few pigs were coprologically positive at slaughter (week 14 p.i.).

\section{Calculation of the phenotypic traits}

All FECs for A. suum and T. suis for each week were used as phenotypic traits in the heritability calculations. For 
FECs of T. suis at weeks 6, 12 and 14 p.i., we were not able to keep the residual kurtosis within normal range despite transformation, and these results were omitted. A number of other ways of describing the phenotypes were included: T. suis FEC weeks $7+8$, T. suis FEC weeks $8+9$ and T. suis FEC weeks 7-9 as the sum of the FECs for these given weeks; T. suis and A. suum total FECs (the areas under the curves) as the sums of the FECs from week 6 to 14 p.i., where FECs weeks 11 and 13 p.i. were calculated as the average of the two flanking weeks; $A$. suum and $T$. suis FEC peaks were defined as the maximum FECs observed for a given pig. In addition, as phenotypes for $A$. suum we also used worm count, A. suum biomass (total weight of the worms obtained from each pig), mean worm length and mean worm weight, being aware that storing in $70 \%$ ethanol may have affected weight and length. Lastly, mean FEC per female $(n=129)$ was calculated as FEC week 14 p.i. divided by the number of females obtained at slaughter.

\section{Statistical analysis}

We performed heritability analysis using a variance components approach and software package SOLAR (Almasy and Blangero, 1998) where all parameter estimates are maximum likelihood estimates, and all hypothesis testing is conducted using likelihood ratio testing. Variance components-based heritability analysis is based on decomposition of the phenotypic variance into the effects of genetic factors, shared environmental factors (for example, using a 'household' model), and individual-specific factors due to the unique environmental exposures and measurement error. The effects of measured covariates (for example, sex) can be included in the analysis as predictors of the phenotypic mean (for example, using a linear regression model). We first estimated the (narrow sense) heritability $\left(h^{2}\right)$, which is the proportion of the total phenotypic variance that can be attributed to the additive effects of genetic factors. We also estimated to which degree two phenotypic traits are under the regulation of overlapping genetic factors, by performing bivariate heritability analysis. The additive genetic correlation $\left(\rho_{\mathrm{G}}\right)$ and the random environmental correlation $\left(\rho_{\mathrm{E}}\right)$ were estimated using SOLAR, and the phenotypic correlation $\left(\rho_{\mathrm{P}}\right)$ was subsequently calculated by:

$$
\rho_{\mathrm{P}}=\sqrt{h_{1}^{2}} \cdot \sqrt{h_{2}^{2}} \cdot \rho_{\mathrm{G}}+\sqrt{\left(1-h_{1}^{2}\right)} \cdot \sqrt{\left(1-h_{2}^{2}\right)} \cdot \rho_{\mathrm{E}}
$$

where $h_{1}^{2}$ is the heritability of trait 1 and $h_{2}^{2}$ is the heritability of trait 2 (Czerwinski et al., 1999). The entire pedigree relationships were used in the analyses. It was assumed that the founding sows and boars were unrelated.

All significant covariates from the univariate analysis were included in the bivariate analysis. Owing to the aggregated distribution of $A$. suum and T. suis within the host population, FECs and worm counts were all transformed with $\ln ($ trait +1$)$ to correct for skewness. FEC per female worm was inverse normal transformed, whereas pig body weight week 0 p.i. and pig weight gain remained untransformed.

For each trait, the heritability was calculated with paddock (1-6) included as shared environmental effect ('household') and body weight at week 0 p.i., sex, farm (1 and 2) and delivery (first or second) as covariates.
A permissive threshold was used including covariates in the final model with $P<0.1$. Significant covariates were included in the bivariate analysis.

The parameter $k$ of the negative binomial distribution was calculated for all $A$. suum and T. suis FEC for each week 7-14 p.i. and $A$. suum worm load at necropsy to determine how levels of aggregation change over time. Decreasing value of $k$ indicates increase in aggregation. $k$ was computed as follows: $k=$ mean $^{2} /$ (variance-mean).

\section{Results}

\section{Descriptive information}

All faecal samples week 0 p. i. were found to be negative and are therefore omitted from the following figures and tables. Approximately two-third of the pigs became positive for $A$. suum and continued to excrete eggs (Figure 1). A peak in prevalence around week 8 p.i. was observed for $T$. suis after which expulsion took place leaving $5 \%$ FEC-positive pigs by week 14 p.i.

Figure 2 shows the aggregation of $A$. suum within the host population with 27 and $22 \%$ of the pigs harbouring $80 \%$ of the total worm population as measured by worm count at necropsy and FEC by week 8 p.i., respectively $(k=0.25$ and 0.32$)$. The geometric mean for worm counts was 8 (all pigs). In infected pigs, the mean intensity of $A$. suum was 29 (s.d. $=46$; minimum-maximum: 1-422). For A. suum FEC weeks 7-14 p.i, the minimum-maximum estimate for $k$ was $0.18-0.4$ as depicted in Figure 1 . As for A. suum, a skewed distribution was also observed for T. suis (Figure 2), with $22 \%$ of the pig population carrying $80 \%$ of the total infection $(k=0.33)$, as measured by FECs at week 8 p.i. The minimum-maximum of $k$ for T. suis FEC weeks 7-14 p.i. was 0.03-0.33 (Figure 1).

\section{Heritability estimates}

The heritabilities for $A$. suum FECs were consistently around 0.3 (all $h^{2}, P<0.002$ ) from week 7 p.i. and onwards (Figure 3 ). In contrast, the heritability estimates for T. suis FECs (all $h^{2}, P<0.001$ ) peaked by week 8 p.i. with $73 \%$ of the phenotypic variation explained by genetic components, where after it declined following the same pattern as the prevalence. T. suis FEC weeks 7 and 8 p.i., T. suis FEC weeks 7-9 p.i., total FEC and peak of egg excretion had heritabilities around 0.62 , whereas for T. suis FEC weeks 8 and 9 p.i. $72 \%$ of the variation could be explained by genetic components (Table 2 ).

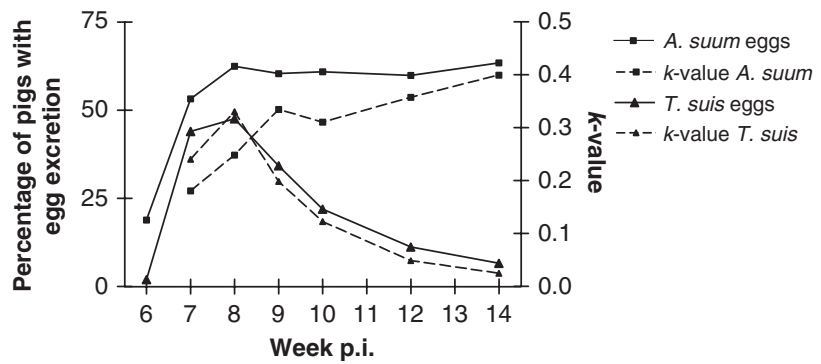

Figure 1 Percentage of $A$. suum and T. suis trickle-infected pigs $(n=195)$ with egg excretion ( $>40$ and $>20$ faecal egg count (FEC) used as cutoffs, respectively) and the respective $k$-values as a function of week p.i. 


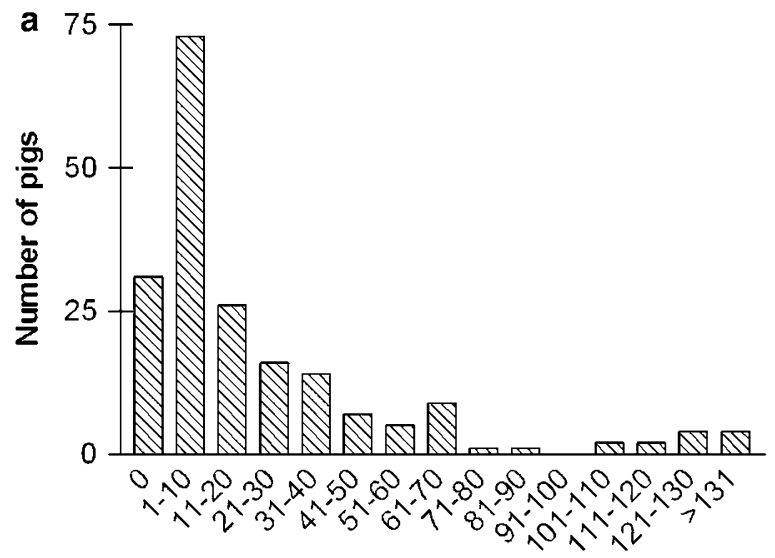

Worm burden

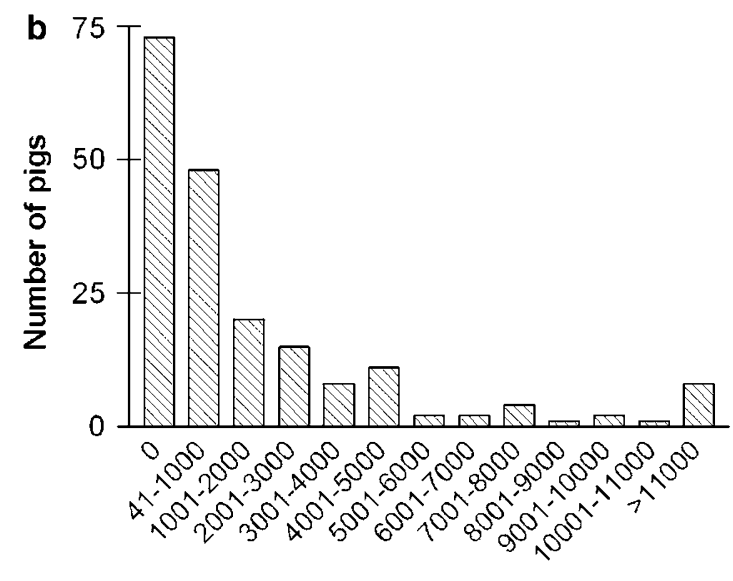

FEC

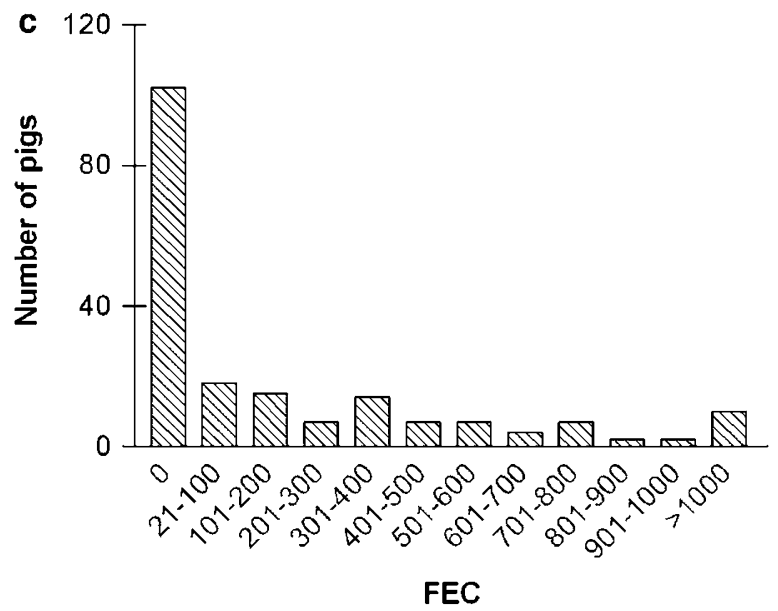

Figure 2 Frequency distribution of $A$. suum and T. suis in trickleinfected pigs $(n=195)$. (a) Worm counts for A. suum at week 14 p.i. (b) Faecal egg count (FEC) for $A$. suum week 8 p.i. (FEC $<41$ was considered 'false positive'). (c) FEC for T. suis at week 8 p.i. $(\mathrm{FEC}=20$ was considered 'false positive').

For A. suum, there was no significant effect of paddock (that is, common effect, $c^{2}$ ) or any of the covariates, accounting for less than $2 \%$ of the variation. Paddock and sex were also found to be nonsignificant for T. suis, whereas delivery and farm had significant $(P<0.05)$ effects on the variance of FECs, with highest figures from

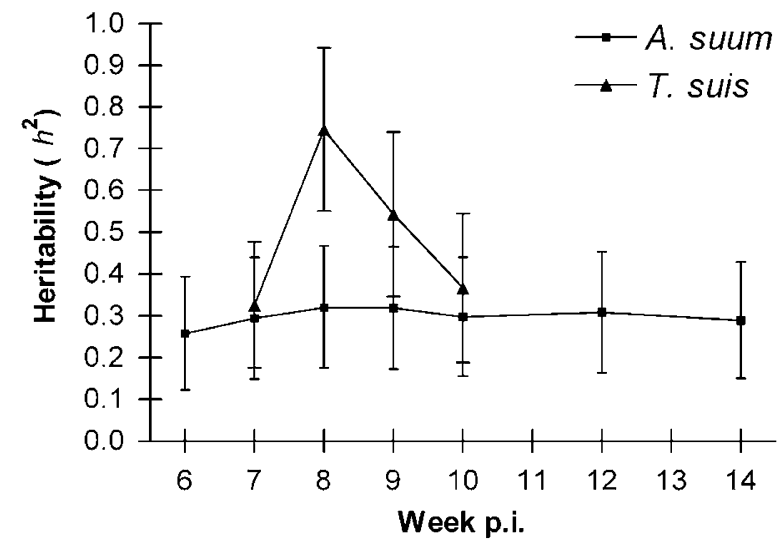

Figure 3 Heritability estimates by week p.i. for A. suum and T. suis faecal egg counts (FECs) in pigs during trickle infections (for all $h^{2}$, $P<0.002$ ). Heritability for Trichuris at weeks 6,12 and 14 p.i. had very skew distribution due to few infected pigs and were omitted. Bars: s.e.

pigs originating from delivery 2 and farm 1, respectively. The significant covariates accounted for $18-26 \%$ of the total variation.

The worm count gave the highest heritability estimate (0.44) obtained for A. suum, whereas total FEC, peak of egg excretion and A. suum worm biomass had heritabilities around 0.35 (Table 3 ). The heritability of mean FEC per female worm was found to be low and nonsignificant. All covariates and the effect of paddock were nonsignificant. Heritabilities of mean length and mean weight of worms, both in total and by sex of worm, were found to be low and nonsignificant (data not shown).

The heritabilities for weight were $0.26(0.13), 0.22(0.12)$ and $0.25(0.12)$ at week 0,7 and 14 p.i., respectively (standard error in brackets) and significant at $P<0.01$. Delivery and sex had significant $(P<0.05)$ effects on weight at week 7 and 14 p.i., respectively.

\section{Bivariate analyses}

High genetic (1.00) and phenotypic correlations (0.810.99 ) were found between $T$. suis traits, and similar results were obtained for $A$. suum with genetic correlations of $0.97-1.00$ and phenotypic correlations of $0.73-$ 1.00 (Table 4). Genetic correlations between A. suum and T. suis traits were all found to be high $(0.75-0.89)$ and significant, with the highest value obtained for $A$. suum FEC and T. suis FEC week 8 p.i. $(P=0.007)$. In contrast, the phenotypic correlations between $A$. suum and T. suis traits were relatively low with estimates from 0.15 to 0.32 . The genetic correlations between the parasiterelated traits and weight gain of the pigs were all negative $(-0.01$ to -0.38$)$ but not significantly different from zero.

Bivariate analysis of worm counts and other traits for A. suum week 14 p.i. showed high genetic and phenotypic correlations (Table 5).

\section{Discussion}

In this experimental infection study, we have found a classical aggregated distribution of $A$. suum $(k=0.18-0.4)$ with approximately $20-30 \%$ of the host population 
Table 2 Heritability estimates for T. suis

\begin{tabular}{|c|c|c|c|c|c|c|}
\hline Trait & $\mathrm{h}^{2}$ & s.e & P-value & P-value for delivery ${ }^{\mathrm{a}}$ & P-value for farm $^{\mathrm{b}}$ & Percentage of variance due to all final covariates \\
\hline FEC weeks 7 and 8 p.i. & 0.61 & 0.19 & $<0.0001$ & 0.02 & 0.001 & 21.2 \\
\hline FEC weeks 8 and 9 p.i. & 0.76 & 0.20 & $<0.0001$ & 0.018 & 0.003 & 22.5 \\
\hline FEC weeks 7-9 p.i. & 0.64 & 0.19 & $<0.0001$ & 0.013 & 0.0008 & 24.3 \\
\hline Total FEC output & 0.64 & 0.19 & $<0.0001$ & 0.007 & 0.0006 & 25.8 \\
\hline Peak value of FEC & 0.64 & 0.19 & $<0.0001$ & 0.009 & 0.0008 & 24.3 \\
\hline
\end{tabular}

Abbreviations: FEC, faecal egg count; s.e., standard error.

a Deliveries 1 and 2.

${ }^{\mathrm{b}}$ Farms 1 and 2.

Table 3 Heritability for $A$. suum

\begin{tabular}{lccc}
\hline Trait & $\mathrm{h}^{2}$ & s.e. & P-value \\
\hline Total FEC output & 0.36 & 0.15 & $<0.0001$ \\
Peak value of FEC & 0.37 & 0.16 & $<0.0001$ \\
Worm count & 0.45 & 0.17 & $<0.0001$ \\
Biomass of worms & 0.35 & 0.15 & $<0.0001$ \\
Mean FEC per female $(n=129)$ & 0.06 & 0.12 & 0.21 \\
\hline
\end{tabular}

Abbreviations: FEC, faecal egg count; s.e., standard error.

carrying $80 \%$ of the worm population as seen in other studies (for example, Boes et al., 1998b). For T. suis FECs in trickle-infected pigs, we have for the first time shown a similar skewed distribution of the infection $(k=0.03-$ 0.33). Eggs can be detected in faeces from around week 6 p.i. for both parasites. After week 6 p.i., the prevalences for both parasites increase. Despite this initial similarity, the population dynamics of the two parasites differ dramatically (Figure 1). The majority of $A$. suum larvae get expelled from the small intestine during the early phase of infection ( $\sim$ week 3 p.i.) where after the small remaining $A$. suum population seems unaffected by host immunity and these worms grow and give rise to the stable $A$. suum FEC around week 8 p.i. and onwards when they are fully mature (Roepstorff et al., 1997). For T. suis, the prevalence drops dramatically at week 8 p.i. (Figure 1), indicating population dynamics similar to single infections with very few FEC positive hosts at week 11 p.i. (Kringel and Roepstorff, 2006). The observed difference in population dynamics between $A$. suum and T. suis based on FEC is therefore related to the time point for the onset of main immune response and the nature of the response mounted by the host.

The heritability estimates for $A$. suum FECs week 7 p.i. onwards were around 0.30 , which means that genetic factors account for $30 \%$ of the variation in FECs. A heritability of 0.35 was obtained when using the phenotype 'total $A$. suum eggs excretion' (area under the curve), which one may argue is a better measure of the phenotype as it covers the whole infection period. However, the highest heritability was obtained using the actual worm counts (0.44). Our heritability estimates are of similar magnitude as the ones estimated for A. lumbricoides in a human population in Nepal, where Williams-Blangero et al. (1999) found that genetics accounted for $30-50 \%$ of the variation in FEC, worm count and worm biomass (estimated after treatment). These findings are mutually encouraging, due to the close genetic relationship between $A$. suum and
A. lumbricoides (Anderson, 2001) and the physiological similarities of the two hosts. In contrast, Ellis et al. (2007) did not find a genetic component to $A$. lumbricoides infections in China but only a household effect accounting for $32 \%$ of the variation. But as mentioned by these authors, this may be due to their use of binary phenotypes (infected versus non-infected) and associated lack of quantitative power, which are important when studying helminth infections (Quinnell, 2003).

Heritability estimates obtained for fecundity measures (for example, number of eggs in uterus) and length of worms have shown to be higher than for actual worm counts in lambs infected with Teladorsagia circumcincta (Stear et al., 1997) and appear to be associated with IgA levels (Stear et al., 1999). In contrast, for A. suum, we have found low and nonsignificant heritabilities both for FEC per female and worm size. This indicates that $A$. suum fecundity may be under the regulation of mechanisms other than worm counts, for example, responding in a different way to host immunity.

In human populations, heritability for T. trichiura infection has been found to be approximately 0.30 (Williams-Blangero et al., 2002; Ellis et al., 2007), with no or low effect of household $(4 \%)$. In contrast, we have found heritability of $0.6-0.7$ for $T$. suis FECs. This difference could reflect the degree of controlled variables in the two studies but could also be due to differences in host reaction. The prevalence for $T$. trichiura infection as measured by egg excretion is generally peaking around the second decade of life for humans and is chronic by nature (Stephenson et al., 2000), whereas the peak is reached early in life for pigs (Roepstorff et al., 1992) or early after exposure followed by expulsion (Figure 1).

In contrast to $A$. suum where there were no significant effects observed for any of the included covariates, pigs from farm 1 and delivery 2 had significant higher T. suis FEC. We do not have any plausible explanation for these discrepancies between farms and deliveries except perhaps that effect of farm must be due to different underlying genetics of the sows used at the two farms.

A caveat in our heritability estimates is, however, that the genetic effect obtained here is confounded with any litter environment effect as the piglets were kept together with the sow for the first 5 weeks of life. A potential litter effect could be due to different levels of nutrition received by piglets in different litters, or more specifically related to transfer of maternal immunity by colostrum or an infection during the suckling period. We consider litter effects due to maternal immunity to be unlikely because all sows were tested negative for $T$. suis and $A$. suum except one for $A$. suum (FEC, 140). In 
Table 4 Genetic $\left(r_{\mathrm{g}}\right.$, above diagonal) and phenotypic correlations ( $r_{\mathrm{p}}$, below diagonal) for some of the phenotypic traits

\begin{tabular}{lcccccccc}
\hline & $\begin{array}{c}\text { T. suis FEC } \\
\text { week 8 p.i }\end{array}$ & $\begin{array}{c}\text { T. suis peak } \\
\text { value of FEC }\end{array}$ & $\begin{array}{c}\text { T. suis total } \\
\text { FEC output }\end{array}$ & $\begin{array}{c}\text { A. suum FEC } \\
\text { week 8 p.i suum peak }\end{array}$ & $\begin{array}{c}\text { A. suum total } \\
\text { value of FEC }\end{array}$ & $\begin{array}{c}\text { A. suum } \\
\text { FEorm count }\end{array}$ & Weight gain \\
worm count & \\
\hline T. suis FEC week 8 p.i & - & $1.00^{* *}$ & $1.00^{* *}$ & $0.89^{* *}(0.21)$ & $0.83^{*}(0.21)$ & $0.83^{*}(0.22)$ & $0.79^{*}(0.22)$ & $-0.01(0.43)$ \\
T. suis peak value of FEC & 0.81 & - & 1.00 & $0.87^{*}(0.27)$ & $0.79^{*}(0.26)$ & $0.79^{*}(0.26)$ & $0.76^{*}(0.27)$ & $-0.10(0.45)$ \\
T. suis total FEC output & 0.82 & 0.99 & - & $0.85^{*}(0.28)$ & $0.78^{*}(0.26)$ & $0.78^{*}(0.27)$ & $0.75^{*}(0.27)$ & $-0.08(0.08)$ \\
A. suum FEC week 8 p.i & 0.25 & 0.15 & 0.15 & - & $0.99^{*}(0.05)$ & $0.99^{*}(0.04)$ & $0.97^{*}(0.07)$ & $-0.19(0.50)$ \\
A. suum peak value of FEC & 0.23 & 0.16 & 0.16 & 0.89 & - & 1.00 & $0.99^{*}(0.06)$ & $-0.38(0.45)$ \\
A. suum total FEC output & 0.24 & 0.15 & 0.15 & 0.89 & 1.00 & - & $0.98^{*}(0.06)$ & $-0.38(0.46)$ \\
A. suum worm count & 0.32 & 0.22 & 0.22 & 0.75 & 0.81 & 0.81 & -0.07 & $-0.02(0.47)$ \\
Weight gain & -0.19 & -0.10 & -0.11 & -0.03 & -0.07 & -0.08 & -0.07 & - \\
\hline
\end{tabular}

Abbreviation: FEC, faecal egg count.

Total egg output, area under the curve.

Standard error in brackets.

${ }^{*} P<0.05,{ }^{* *} P<0.01$.

Table 5 Genetic $\left(r_{\mathrm{g}}\right)$ and phenotypic $\left(r_{\mathrm{p}}\right)$ correlations for A. suum worms at slaughter

\begin{tabular}{lllll}
\hline & $\mathrm{r}_{g}$ & s.e. & $\mathrm{P}_{g}$ & $\mathrm{r}_{p}$ \\
\hline Worm burden, A. suum FEC week 14 p.i. & 1 & - & 0.07 & 0.86 \\
Worm burden, A. suum biomass & 1 & - & 0.03 & 0.96 \\
A. suum FEC week 14 p.i., A. suum biomass & 1 & - & 0.19 & 0.90 \\
\hline
\end{tabular}

Abbreviations: FEC, faecal egg count; s.e., standard error.

addition, all pigs were initially (week 0 p.i.) FEC negative.

Genetic correlation close to one was found between Haemonchus contortus and Trichostrongylus colubriformis infection levels in sheep (Gruner et al., 2004). We have also found evidence for genetic correlation between A. suum and T. suis infections (0.75-0.89), suggesting that some of the same genes may play a role in the regulation of the worm loads. This observation is interesting when considering the different population dynamics of the two parasites (Figure 1). The majority of the pigs are able to completely expel all of the T. suis (Kringel and Roepstorff, 2006) and hinder the establishment of new incoming worms (unpublished data), whereas a stable parasite population situation seems to be established for $A$. suum. Correlation between $A$. lumbricoides and T. trichiura in the human population is normally explained by similar transmission routes and egg biology, but may also be due to the regulation of some of the same genes as our finding in the pig suggest. However, Williams-Blangero et al. (2008a,b) did not identify overlapping quantitative trait loci for A. lumbricoides and T. trichiura infections in humans, suggesting that different genes are involved here, even though the population dynamics and prevalences of the two parasites show similar patterns in the human population (for example, Anderson et al., 1993).

As the high heritability for T. suis is linked to the short period where the prevalence is peaking (week 8 p.i.), this makes the identification of high- and low-responder pigs more problematic than for A. suum (for example, if high responder pigs should be identified for breeding). For T. suis, exposure period needs to be known to predict when FEC is peaking. It would therefore be beneficial, if a more 'stable' phenotype (for example, an ELISA) could be used to identify high- and low-responder T. suis pigs.
For example, Davies et al. (2005) found high genetic correlations between a range of indicator traits and nematode infections in lambs, and this could aid in the selection for increased resistance to gastrointestinal nematodes. Douch et al. (1995) have shown that in sheep infected with $T$. colubriformis, selection based on serum antibodies would result in $51-67 \%$ of the genetic gain in FEC compared with that obtained by FEC selection directly. However, as mentioned by these authors, FEC in itself is an indirect measure of worm number and antibody titre may be as good or a better measure of a host's ability to resist parasite infection.

In naturally infected sheep, a negative genetic correlation has been described between FEC and weight gain, suggesting that resistant animals have a higher growth rate (Bishop et al., 1996; Bouix et al., 1998; Gauly and Erhardt, 2001). We have also found a negative correlation between $A$. suum and T. suis FECs and weight gain. However, this correlation was not different from zero, suggesting that selection for more A. suum- and T. suisresistant pig will not influence the growth performance in this breed of pigs.

Although aggregation and predisposition of A. suum within a pig population previously have been shown in single and trickle infections (Roepstorff et al., 1997; Boes et al., 1998b), the present study for the first time demonstrates the role of host genetic. Our findings suggest that future whole genome scan would be useful to identify regions and specific genes, which are involved in the regulation of both $A$. suum and T. suis infection levels in pigs. Genetic markers may be useful in breeding programs (for example, marker-assisted selection) and thereby production of more parasite-resistant pigs or in choosing 'the right animal for the right purpose'. In addition, if specific genes can be identified these would undoubtedly enhance our understanding of the host immunological response to infection with these parasites in the pig and with related species in humans.

\section{Acknowledgements}

The help of Frederik Andersen, Niels Peter K Hansen, Trine Andersen, Margrethe Pearman, Anders Rasmussen, Henrik B Lassen, Maria Langkjær, Peter Kristiansen and Niels Midtgaard is greatly appreciated. A big thanks to Jack Kent Jr and Tom Dyer at SFBR for help with statistical issues and SOLAR analysis. We thank Sarah 
Williams-Blangero for an unforgettable stay at SFBR. The Danish National Research Foundation is acknowledged for the financial support of this project under the auspices of the Danish Centre for Experimental Parasitology.

\section{References}

Almasy L, Blangero J (1998). Multipoint quantitative-trait linkage analysis in general pedigrees. Am J Hum Genet 62: 1198-1211.

Anderson RM, May RM (1985). Helminth infections of humans-mathematical models, population dynamics, and control. Adv Parasitol 24: 1-101.

Anderson TJC (2001). The dangers of using single locus markers in parasite epidemiology: Ascaris as a case study. Trends Parasitol 17: 183-188.

Anderson TJC, Zizza CA, Leche GM, Scott ME, Solomons NW (1993). The distribution of intestinal helminth infections in a rural village in Guatemala. Mem Inst Oswaldo Cruz 88: 53-65.

Bishop SC, Bairden K, McKellar QA, Park M, Stear MJ (1996). Genetic parameters for faecal egg count following mixed, natural, predominantly Ostertagia circumcincta infection and relationships with live weight in young lambs. Anim Sci 63: 423-428.

Boes J, Johansen MV, Eriksen L, Bogh HO, Nansen P, Stephenson LS (1998a). False-positive Trichuris suis egg counts in pigs in relation to coprophagia. Parasite 5: 91-93.

Boes J, Medley GF, Eriksen L, Roepstorff A, Nansen P (1998b). Distribution of Ascaris suum in experimentally and naturally infected pigs and comparison with Ascaris lumbricoides infections in humans. Parasitology 117: 589-596.

Boes J, Nansen P, Stephenson LS (1997). False-positive Ascaris suum egg counts in pigs. Int J Parasitol 27: 833-838.

Bouix J, Krupinski J, Rzepecki R, Nowosad B, Skrzyzala I, Roborzynski M et al. (1998). Genetic resistance to gastrointestinal nematode parasites in Polish long-wool sheep. Int J Parasitol 28: 1797-1804.

Chan L, Bundy DAP, Kan SP (1994a). Aggregation and predisposition to Ascaris lumbricoides and Trichuris trichiura at the familial level. Trans $R$ Soc Trop Med Hyg 88: 46-48.

Chan L, Bundy DAP, Kan SP (1994b). Genetic relatedness as a determinant of predisposition to Ascaris lumbricoides and Trichuris trichiura infection. Parasitol 108: 77-80.

Czerwinski SA, Mahaney MC, Williams JT, Almasy L, Blangero J (1999). Genetic analysis of personality traits and alcoholism using a mixed discrete continuous trait variance component model. Genet Epidemiol 17(Suppl 1): S121-S126.

Davies G, Stear MJ, Bishop SC (2005). Genetic relationships between indicator traits and nematode parasite infection levels in 6 month old lambs. Anim Sci 80: 143-150.

Dekkers JCM (2004). Commercial application of marker- and gene-assisted selection in livestock: strategies and lessons. I Anim Sci 82: E313-E328.

Douch PGC, Green RS, Morris CA, Bisset SA, Vlassoff A, Baker RL et al. (1995). Genetic and phenotypic relationships among anti-Trichostrongylus colubriformis antibody level, fecal egg count and body-weight traits in grazing Romney sheep. Livest Prod Sci 41: 121-132.

Ellis MK, Raso G, Li YS, Rong Z, Chen HG, McManus DP (2007). Familial aggregation of human susceptibility to $\mathrm{co}^{-}$and multiple helminth infections in a population from the Poyang Lake region, China. Int J Parasitol 37: 1153-1161.

Eriksen L, Nansen P, Roepstorff A, Lind P, Nilsson O (1992). Response to repeated inoculations with Ascaris suum eggs in pigs during the fattening period. I. Studies on worm population kinetics. Parasitol Res 78: 241-246.
Forrester JE, Scott ME, Bundy DA, Golden MH (1990). Predisposition of individuals and families in Mexico to heavy infection with Ascaris lumbricoides and Trichuris trichiura. Trans R Soc Trop Med Hyg 84: 272-276.

Gauly M, Erhardt G (2001). Genetic resistance to gastrointestinal nematode parasites in Rhon sheep following natural infection. Vet Parasitol 102: 253-259.

Gruner L, Bouix J, Brunel JC (2004). High genetic correlation between resistance to Haemonchus contortus and to Trichostrongylus colubriformis in INRA 401 sheep. Vet Parasitol 119: $51-58$.

Jørgensen CB, Cirera S, Archibald A, Andersson L, Fredholm M, Edfors-Lilja I (2004). Porcine polymorphisms and methods for detecting them. International application published under the patent cooperation treaty (PCT). PCT/DK2003/ 000807 or WO2004/048606 A2.

Kringel H, Roepstorff A (2006). Trichuris suis population dynamics following a primary experimental infection. Vet Parasitol 139: 132-139.

Larsen MN, Roepstorff A (1999). Seasonal variation in development and survival of Ascaris suum and Trichuris suis eggs on pastures. Parasitol 119: 209-220.

Meijerink E, Fries R, Vogeli P, Masabanda J, Wigger G, Stricker $C$ et al. (1997). Two alpha(1,2) fucosyltransferase genes on porcine chromosome $6 \mathrm{q} 11$ are closely linked to the blood group inhibitor (S) and Escherichia coli F18 receptor (ECF18R) loci. Mamm Genome 8: 736-741.

Milan D, Jeon JT, Looft C, Amarger V, Robic A, Thelander M et al. (2000). A mutation in PRKAG3 associated with excess glycogen content in pig skeletal muscle. Science 288: 1248-1251.

Oksanen A, Eriksen L, Roepstorff A, Ilsoe B, Nansen P, Lind P (1990). Embryonation and infectivity of Ascaris suum eggs-a comparison of eggs collected from worm uteri with eggs isolated from pig feces. Acta Vet Scand 31: 393-398.

Quinnell RJ (2003). Genetics of susceptibility to human helminth infection. Int J Parasitol 33: 1219-1231.

Roepstorff A (2003). Ascaris suum in Pigs: Population Biology and Epidemiology. Danish Centre for Experimental Parasitology, Royal Veterinary and Agricultural University: Copenhagen, Denmark

Roepstorff A, Eriksen L, Slotved HC, Nansen P (1997). Experimental Ascaris suum infection in the pig: worm population kinetics following single inoculations with three doses of infective eggs. Parasitol 115: 443-452.

Roepstorff A, Jørgensen RJ, Nansen P, Henriksen SA, Skovgaard-Petersen J, Andreasen M (1992). Parasitter hos økologiske svin (in Danish). Landsudvalget for Svin, Danske Slagterier: Copenhagen, 1-36.

Roepstorff A, Murrell KD (1997). Transmission dynamics of helminth parasites of pigs on continuous pasture: Ascaris suum and Trichuris suis. Int J Parasitol 27: 563-572.

Roepstorff A, Murrell KD (1998). The Epidemiology, Diagnosis and Control of Helminth Parasites in Swine. A FAO handbook. FAO: Rome, Italy.

Sayers G, Good B, Hanrahan JP, Ryan M, Angles JM, Sweeney T (2005). Major histocompatibility complex DRB1 gene: its role in nematode resistance in Suffolk and Texel sheep breeds. Parasitol 131: 403-409.

Stear MJ, Bairden K, Bishop SC, Buitkamp J, Duncan JL, Gettinby $G$ et al. (1997). The genetic basis of resistance to Ostertagia circumcincta in lambs. Vet J 154: 111-119.

Stear MJ, Strain S, Bishop SC (1999). How lambs control infection with Ostertagia circumcincta. Vet Immunol Immunopathol 72: 213-218.

Stephenson LS, Holland CV, Cooper ES (2000). The public health significance of Trichuris trichiura. Parasitol 121: S73-S95.

Van Laere AS, Nguyen M, Braunschweig M, Nezer C, Collette C, Moreau L et al. (2003). A regulatory mutation in IGF2 causes a major QTL effect on muscle growth in the pig. Nature 425: 832-836. 
Williams-Blangero S, McGarvey ST, Subedi J, Wiest PM, Upadhayay RP, Rai DR et al. (2002). Genetic component to susceptibility to Trichuris trichiura: evidence from two Asian populations. Genet Epidemiol 22: 254-264.

Williams-Blangero S, Subedi J, Upadhayay RP, Manral DB, Rai DR, Jha B et al. (1999). Genetic analysis of susceptibility to infection with Ascaris lumbricoides. Am J Trop Med Hyg 60: 921-926.
Williams-Blangero S, VandeBerg JL, Subedi J, Jha B, CorreaOliveira R, Blangero J (2008a). Localization of multiple quantitative trait loci influencing susceptibility to infection with Ascaris lumbricoides. I Infect Dis 197: 66-71.

Williams-Blangero S, VandeBerg JL, Subedi J, Jha B, Dyer T, Blangero J (2008b). Two quantitative trait loci influence whipworm (Trichuris trichiura) infection in a Nepalese population. J Infect Dis 197: 1198-1203. 